Ger J Exerc Sport Res 2020 · 50:406-416 https://doi.org/10.1007/s12662-020-00661-y Eingegangen: 26. Juni 2019

Angenommen: 21. Mai 2020

Online publiziert: 16 . Juni 2020

(c) Der/die Autor(en) 2020

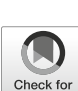

\author{
Herbert Woratschek ${ }^{1} \cdot$ Mario Kaiser $^{1} \cdot$ Christian Durchholz $^{2} \cdot$ Tim Ströbel $^{3}$ \\ 'Chair in Marketing and Service Management, University of Bayreuth, Bayreuth, Deutschland \\ ${ }^{2}$ bbg Betriebsberatungs $\mathrm{GmbH}$, Bayreuth, Deutschland \\ ${ }^{3}$ Professorship in Marketing \& Sport Management, University of Bayreuth, Bayreuth, Deutschland
}

\title{
Preisaufschläge für die sportliche Relevanz
}

\section{Eine Conjoint-Analyse für Tickets von Sportevents}

lerdings die Akzeptanz der Fans für das Sportevent gefährden. Zu geringe Ticketpreise riskieren auf der anderen Seite eine nicht ausreichende Finanzierung des Sportevents und damit der Sportteams. Im Idealfall erreicht man optimale Erlöse bei einer vollen Stadionauslastung und möglichst hohen, von den Käufern akzeptierten Ticketpreisen. Volle Stadien erhöhen zudem das Interesse der Medien und ermöglichen höhere Medieneinnahmen, aber auch höhere Vermarktungserlöse im Sponsoring. Die Preisgestaltung für Tickets von Sportevents ist somit von elementarer Bedeutung. Die Ticketpreise sollten daher den Präferenzen und Preisbereitschaften der potenziellen Zuschauer entsprechen.

Dennoch werden in den meisten Sportorganisationen Ticketpreise auf Grundlage von Daumenregeln oder gar dem Bauchgefühl von Entscheidern festgelegt. Insbesondere besteht im Sportmanagement Forschungsbedarf in Bezug auf Sportarten und Events, bei denen keine historischen Daten über die Präferenzen und Preisbereitschaften der potenziellen Eventbesucher vorliegen. Dies trifft insbesondere für Einzelsportevents im Vergleich zu regelmäßig stattfindenden Ligaspielen zu. Ebenso besteht Forschungsbedarf in Bezug auf die Preisbereitschaft von Zuschauern für Sportevents mit erhöhter sportlicher Relevanz.

Die sportliche Relevanz bezeichnet dabei den Grad der Bedeutung eines sportlichen Wettbewerbes für Zuschau- er, Medien und Teams oder andere Akteure. So hat zum Beispiel ein Spiel zwischen denselben Mannschaften in unterschiedlichen Wettbewerben eine unterschiedliche sportliche Relevanz, je nachdem, ob es sich beispielsweise um ein Weltmeisterschaftsfinale oder ein Freundschaftsspiel handelt. Von unterschiedlicher Relevanz sind auch Spiele zwischen denselben Mannschaften, je nachdem, ob es innerhalb einer Wettbewerbsserie um die Meisterschaft, einen Mittelfeldplatz oder den Abstieg geht (Woratschek \& Schafmeister, 2008). Somit wird die sportliche Relevanz wie folgt definiert: Die sportliche Relevanz bezeichnet die unterschiedliche gesellschaftliche Stellung eines singulären sportlichen Wettbewerbs (z. B. Meisterschaftsfinale, Spiel um Auf- und Abstieg) oder einer Wettbewerbsserie (z. B. Weltmeisterschaften, Olympische Spiele, Vorbereitungsturniere).

Eine Option zur Ermittlung dieser Präferenzen und Preisbereitschaften könnte darin bestehen, potenzielle $\mathrm{Zu}$ schauer direkt $\mathrm{zu}$ fragen, wie viel sie für ein bestimmtes Ticket bereit sind zu bezahlen. Die direkte Befragung liefert jedoch nur unzureichend valide Ergebnisse (Walker \& Mondello, 2007). Eine weitere Option sind dekompositionelle Methoden wie die ConjointAnalyse. Dabei äußern Probanden ihre Präferenzen in Form von Globalurteilen unterschiedlicher Produkt- oder Dienstleistungskombinationen. Die ConjointAnalyse ist insbesondere zur Erfassung 
des Trade-offs zwischen Preis und Qualitätsmerkmalen von Produkten und Dienstleistungen geeignet. Sie wird daher häufig bei der Gestaltung innovativer Produkte oder Dienstleistungen sowie der Bestimmung von Preisbereitschaften eingesetzt (Backhaus, Erichson, Plinke, \& Weiber, 2018; Baier \& Brusch, 2009a). Dieser innovative Charakter trifft insbesondere auf Events zu, bei denen keine Vergangenheitsdaten vorliegen. Im Sportmanagement wurden mittels Conjoint-Analyse unter anderem die optimale Ausgestaltung von Merchandising-Artikeln (Lee \& Ferreira, 2011) oder Fanclubmitgliedschaften (Theysohn, Hinz, Nosworthy, \& Kirchner, 2009) untersucht. Für die präferenzorientierte Gestaltung von Sporteventtickets verspricht die Conjoint-Analyse daher ebenfalls wertvolle Hinweise.

Die Heterogenität von Zuschauern hinsichtlich ihrer soziodemografischen und psychografischen Merkmale ist hinlänglich bekannt und häufig diskutiert (Hunt, Bristol, \& Bashaw, 1999; Wann \& Branscombe, 1990). Darüber hinaus unterscheiden sich Sportzuschauer auch in Bezug auf ihre Präferenzen und Preisbereitschaften (Mullin, Hardy, \& Sutton, 2014), sodass hier eine Benefit-Segmentierung vorgenommen wird (Mühlbacher \& Botschen, 1990). Die Berücksichtigung der Heterogenität von Zuschauerpräferenzen und Preisbereitschaften mittels einer Clusteranalyse ist somit auch für die Conjoint-Analyse zu gewährleisten. Vor diesem Hintergrund diskutiert die nachfolgende Studie die Eignung der Conjoint-Analyse zur Bestimmung von heterogenen Zuschauerpräferenzen und Preisbereitschaften für Sporteventtickets. Zudem werden die Präferenzen und Preisbereitschaften von Zuschauern im deutschen Frauenfußball im Rahmen eines ConjointExperiments untersucht und anschließend mittels Clusteranalyse in unterschiedliche Segmente unterteilt, um Preisaufschläge (und ggf. Preisabschläge) für relevante Ticketmerkmale, wie die sportliche Relevanz, zu bestimmen. Der Beitrag orientiert sich an folgenden Forschungsfragen:

1. Inwiefern ist die Conjoint-Analyse geeignet, um Ticketpräferenzen und
Preisbereitschaften von Zuschauern für Sportevent-Tickets zu ermitteln?

2. Wie lassen sich Zuschauer von unregelmäßig stattfindenden Veranstaltungen hinsichtlich ihrer Präferenzen segmentieren und wie können diese Segmente beschrieben werden?

3. Wie unterscheidet sich die Preisbereitschaft der identifizierten Zuschauersegmente für verschiedene Ticketangebote hinsichtlich der sportlichen Relevanz und der Sitzqualität?

Untersuchungskontext dieser Studie sind Länderspiele im Frauenfußball. Die Studie liefert zum einen wertvolle Erkenntnisse für Events, die in ihrem Zielmarkt erstmalig stattfinden. Zum anderen bietet sie Orientierung für Sportarten, bei denen nur unzureichende Daten zur Zahlungsbereitschaft von Zuschauern aus der Vergangenheit vorliegen.

\section{Methodische Grundlagen der Conjoint-Analyse}

Diese Studie verfolgt das Ziel, die Präferenzen und Preisbereitschaften von $\mathrm{Zu}$ schauern bei unregelmäßig stattfindenden Sportevents zu identifizieren und die Zuschauer auf Basis dessen in Segmente $\mathrm{zu}$ unterteilen. Insbesondere bei Spielen der deutschen Frauen FußballNationalmannschaft mangelt es an Erfahrungswerten und Vergangenheitsdaten über die Ticketpräferenzen von $\mathrm{Zu}$ schauern. Um die Präferenzen der $\mathrm{Zu}$ schauer und deren Preissensitivität für Sporttickets zu untersuchen, wird hier die traditionelle Conjoint-Analyse in Kombination mit einer Cluster-Analyse eingesetzt. Dekompositionelle Methoden wie die Conjoint-Analyse werden seit den frühen 1970er Jahren in breitem Umfang genutzt, um Trade-offs von Konsumenten zwischen Produkten und Dienstleistungen mit mehreren Merkmalen zu messen (Green \& Rao, 1971; Green \& Srinivasan, 1990; Johnson, 1974). Voraussetzung für die Conjoint-Analyse ist, dass Kunden ihre Produktbewertung anhand verschiedener Eigenschaften mit unterschiedlichen Ausprägungen treffen. Eine Produktkarte in der Conjoint-Analyse besteht demnach aus einem Bündel von Eigenschaften, die wiederum verschie- dene Ausprägungen besitzen (Backhaus et al., 2018). Der Vorteil der ConjointAnalyse für die Bestimmung von Präferenzen und Preisbereitschaften besteht in der Vermeidung einer direkten Frage nach maximalen Ticketpreisen oder den Wichtigkeiten einzelner Merkmale. Die Validität direkter Befragungen wird ohnehin kritisch diskutiert (Walker \& Mondello, 2007). Durch die indirekte Befragung in der Conjoint-Analyse werden die Ergebnisse nicht durch sozial erwünschte Antworten beeinflusst. Aus Managersicht kann die Conjoint-Analyse helfen, die am meisten präferierten Merkmale von Produkten oder Dienstleistungen zu bestimmen. In Kombination mit einer Clusteranalyse ist dies auch für Segmente mit unterschiedlichen Präferenzen möglich.

Die Vorhersage menschlichen Beurteilungs- und Entscheidungsverhaltens stellt das generelle Ziel der ConjointAnalyse dar. Die häufigste Anwendung ist die Preisfindung sowie die Marktsegmentierung. Zudem wird die ConjointAnalyse häufig bei innovativen Produkten oder Dienstleistungen eingesetzt, da für die Ermittlung von Präferenzen und Preisbereitschaften keine Vergangenheitsdaten erforderlich sind (Baier \& Brusch, 2009a). Die zentrale Grundannahme besteht darin, dass das $\mathrm{zu}$ untersuchende Angebot aus einem Bündel von nutzenstiftenden Merkmalen besteht. So werden bei der ConjointAnalyse Produktkarten erzeugt, die von den Probanden ganzheitlich beurteilt und in eine Präferenzreihenfolge gebracht werden; die meist präferierte Produktkarte wird auf Rang eins einsortiert, die am wenigsten präferierte auf dem letzten Rang. Dabei wird angenommen, dass die Präferenzreihenfolge zukünftige Kaufentscheidungen zutreffend antizipiert. Die gesamtheitliche Beurteilung der Produktkarten entspricht demnach einer realistischen Kaufentscheidung, die durch die optische Ausgestaltung der Produktkarten unterstrichen wird (Leigh, MacKay, \& Summers, 1984). Die hier verwendete traditionelle Conjoint-Analyse ist dabei in der Lage, Preisdifferenzen (Preisaufschläge bzw. Preisabschläge) zwischen relevanten Ticketmerkmalen zu bestimmen. 
Darüber hinaus wurden zahlreiche Weiterentwicklungen der Conjoint-Analyse in die Marketingliteratur eingeführt. Diese reichen von der Limit-ConjointAnalyse, über auswahlbasierte Verfahren (z.B. Choice-based Conjoint-Analyse) bis hin zu hybriden Verfahren (z. B. Adaptive Choice-based Conjoint-Analyse). Diese weisen teilweise höhere Validitätspotenziale auf sowie die Möglichkeit, mehr Merkmale in das Forschungsdesign aufzunehmen. Fiedler, Kaltenborn, Lanwehr, und Melles (2017) zeigen eine Übersicht über die spezifischen Vorund Nachteile ausgewählter Varianten. Auf den ersten Blick scheinen die moderneren Varianten der traditionellen Conjoint-Analyse überlegen. Dabei ist darauf hinzuweisen, dass adaptive Varianten der Conjoint-Analyse (z. B. ACA oder $\mathrm{ACBC}$ ) aufgrund der Anpassung der Folgefragen auf die Antworten des Probanden ohne technische Hilfsmittel nicht durchführbar sind. Auch bei einer auswahlbasierten Conjoint-Analyse sind die Reduzierung des Designs und die Auswahl der Choice-Tasks an komplexe Algorithmen gekoppelt, die offline und ohne Computerunterstützung nur sehr schwer durchführbar sind.

Im Hinblick auf die besondere Erhebungssituation dieser Studie muss die Umsetzbarkeit während der Befragungssituation bedacht werden. In der vorliegenden Studie wurden Probanden im Rahmen eines Stadionbesuchs zu ihren Präferenzen befragt. Eine Offline-Befragung durch neuere Conjoint-Varianten hätte eine umfassende technische Ausstattung für alle Interviewer erfordert, was bei der Erhebungssituation und dem zur Verfügung stehenden Erhebungszeitraum große Herausforderungen mit sich gebracht hätte. Darüber hinaus bringt gerade die traditionelle Conjoint-Analyse einen großen Vorteil mit sich. Die hier verwendeten Produktkarten ähneln zum Großteil den von den Probanden genutzten Eventtickets und weisen damit eine sehr hohe Realitätsnähe auf (• Abb. 1). In computergestützten Befragungen (z. B. Sawtooth Software) ist diese Realitätsnähe bei adaptiven Verfahren gar nicht und bei auswahlbasierten Varianten nur mit einem massiven Aufwand darstellbar.

Ger J Exerc Sport Res 2020 · 50:406-416 https://doi.org/10.1007/s12662-020-00661-y

(c) Der/die Autor(en) 2020

\section{H. Woratschek · M. Kaiser · C. Durchholz · T. Ströbel}

\section{Preisaufschläge für die sportliche Relevanz. Eine Conjoint-Analyse für Tickets von Sportevents}

\section{Zusammenfassung}

Für die Finanzierung von Sportevents ist das Ticketing eine zentrale Einnahmequelle. Es liegen in diesem Bereich jedoch weder in Praxis noch Forschung valide Erfahrungsdaten vor. Der vorliegende Beitrag greift diese Forschungslücke auf und identifiziert die Bereitschaft von Sporteventzuschauern für Preisaufschläge (bzw. -abschläge) im Rahmen von Wettbewerben mit unterschiedlicher sportlicher Relevanz in unterschiedlichen Sitzkategorien am Beispiel des Frauenfußballs. Der Beitrag zeigt auf, dass die Conjoint-Analyse eine geeignete Methode ist, um Preisbereitschaften für Sportevents in Abhängigkeit ihrer sportlichen Relevanz zu schätzen, insbesondere für Sportevents für die bislang noch keine Erfahrungswerte aus der Vergangenheit vorliegen. Auf Basis einer Befragung von 278
Stadionbesuchern eines Spiels der deutschen Frauen Fußball-Nationalmannschaft zeigt sich, dass die Präferenzen der Zuschauer sehr unterschiedlich sind. Für die Hälfte der Probanden ist die sportliche Relevanz des Wettbewerbs der dominierende Einflussfaktor auf die Preisbereitschaft. Diese sind bereit, einen hohen Preisaufschlag für sportlich relevante Wettbewerbe in Kauf zu nehmen. Die Ergebnisse helfen Sportmanagern, ihre Preisstrategien zu optimieren und gleichzeitig an den Präferenzen und Preisbereitschaften der Zuschauer auszurichten.

\section{Schlüsselwörter}

Preisbereitschaft · Preisgestaltung von Tickets . Innovative Sportevents · Frauenfußball . Conjoint-Analyse

\section{Price premiums for sporting relevance. A conjoint analysis for sport event tickets}

\section{Abstract}

Ticketing is a central income source for financing sport events. However, there is no valid empirical data in this area, neither in practice nor in research. The article addresses this research gap and identifies sport event spectators' willingness to pay of price premiums (or discounts) for matches with varying sporting relevance and different seat categories. For this purpose, an empirical study in women's football serves as research context. The research demonstrates that conjoint analysis is a suitable method for estimating the willingness to pay for sport events depending on the sporting relevance; especially, for sport events that cannot refer to previous data. Based on a survey of 278 stadium visitors of a match of the German women's national football team, it could be shown that spectators' preferences are very heterogenous. For half of the respondents, the sporting relevance of the match is the dominant factor influencing their willingness to pay. They are willing to accept a high price premium for matches with a high sporting relevance. The results help sports managers to optimise their pricing strategies and align them simultaneously with spectators' preferences and willingness to pay.

\section{Keywords}

Willingness to pay · Ticket pricing · Innovative sport events - Women's football - Conjoint analysis

\section{Eignung der Conjoint- Analyse zur Ermittlung von Preisbereitschaften für Sporteventtickets}

Neben den genannten Vorteilen der Conjoint-Analyse sind die Anwendungsmöglichkeiten der Conjoint-Analyse aufgrund ihres dekompositionellen Charakters jedoch auch an unterschiedli- che Bedingungen geknüpft. Woratschek $(1998,2000)$ hat diese für Sportdienstleistungen kritisch diskutiert. Die Erfüllung der nachfolgenden Bedingungen wird für den Einsatz der Conjoint-Analyse zur Ermittlung von Preisbereitschaften für Sporteventtickets als elementar erachtet (Woratschek, 1998, 2000): 


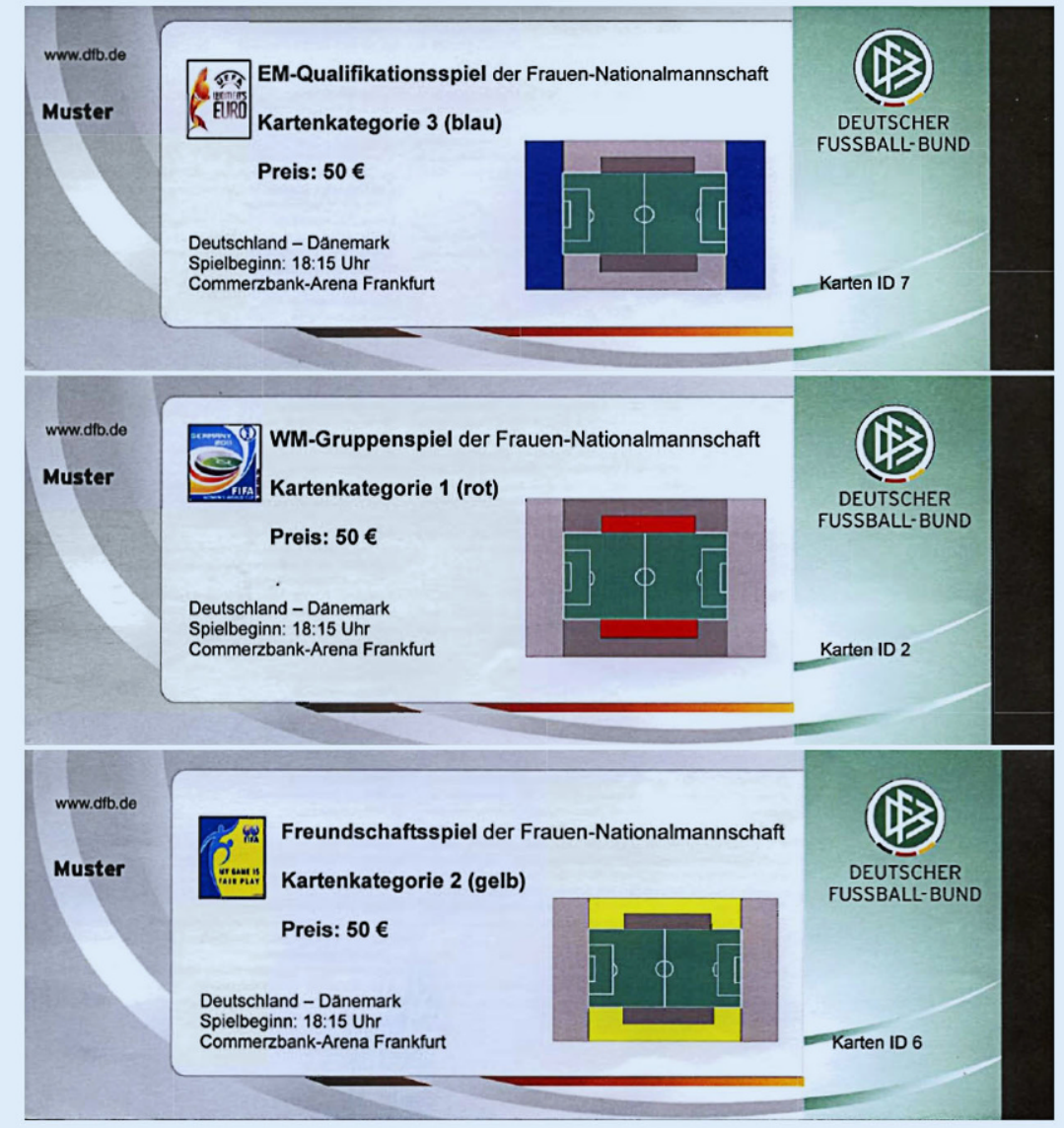

Abb. 1 B Beispielhaftes Design der Produktkarten

\section{Tickets aus dienstleistungs- theoretischer Sicht}

Sportdienstleistungen lassen sich mit Hilfe der Konstrukte Verhaltensunsicherheit (Risikoebene), Integrativität (Prozessebene) und Individualität (Ergebnisebene) charakterisieren (Woratschek, 2000). Die Conjoint-Analyse ist dann für Preisentscheidungen geeignet, wenn die Verhaltensunsicherheit, die Integrativität und die Individualität der Dienstleistung als gering eingestuft werden können. Die Verhaltensunsicherheit beschreibt dabei das empfundene Risiko über das zukünftige Verhalten der Vertragspartner zum Zeitpunkt des Kaufvertrags. Die Integrativität steht für das Ausmaß externer Produktionsfaktoren im Erstellungsprozess einer Sportdienstleistung. Die Individualität beschreibt, inwieweit das Leistungsergebnis maßgeschneidert bzw. standardisiert ist.
Die Verhaltensunsicherheit ist ein zentrales Konstrukt der Informationsökonomik und entsteht aufgrund einer asymmetrischen Informationsverteilung beteiligter Vertragspartner bei Vertragsabschluss. Es ist also unklar, ob die eigenen Erwartungen bei Vertragsabschluss aufgrund möglicher versteckter Absichten oder gar versteckter Handlungen des Vertragspartners wirklich erfüllt werden. So können aus genannten Gründen beispielsweise weder der Verlauf noch das Endresultat einer Physiotherapie vorhergesagt werden. Bei hoher Verhaltensunsicherheit müsste das Risiko endogenisiert werden, wohingegen die Conjoint-Analyse Risiken als exogene Variablen betrachtet. Zudem stehen unspezifische Erwartungen im Widerspruch zu einer exakten Abwägung der Eigenschaften im Experiment. Der Verkauf von Tickets für Sportevents findet in regelmäßigen Abständen statt. Aufgrund der ständigen nachvollzieh- baren Wiederholung der Dienstleistung in einem vergleichbaren Kontext ist die Verhaltensunsicherheit hinsichtlich des Ablaufs eines Sportevents für mögliche Zuschauer als eher gering einzustufen, wenn man vom Verlauf und Ergebnis des sportlichen Wettbewerbs absieht. Aus Perspektive der Verhaltensunsicherheit ist daher die Conjoint-Analyse für die Preisanalyse von Tickets grundsätzlich geeignet.

Die Integrativität bezieht sich auf die Einbeziehung des Kunden als externen Faktor in den Dienstleistungsprozess. Eine hohe Integrativität liegt vor, wenn der Kunde in viele Stufen der Wertschöpfungskette eingreift (Eingriffstiefe) und mit dem Kunden bei der Leistungserstellung häufig und intensiv kommuniziert werden muss (Eingriffsintensität) (Engelhardt, Kleinaltenkamp, \& Reckenfelderbäumer, 1993). Dies ist beispielsweise bei einer Physiotherapie der Fall. Eine hohe Integrativität erfordert daher die Modellierung vieler Merkmalsausprägungen in den unterschiedlichen Stufen der Leistungserstellung. Eine zu hohe Anzahl von relevanten Merkmalen gefährdet die Validität einer Conjoint-Analyse. Zudem entscheidet in der Akquisephase oft die Reputation des Anbieters darüber, welche Dienstleistung ein Nachfrager kauft. Daher ist es fraglich, ob bei einer hohen Integrativität die Faktoren der bei der klassischen Conjoint-Analyse unterstellten Abwägung einzelner Produktmerkmale überhaupt stattfindet und nicht etwa durch ein aus der Reputation abgeleitetes Pauschalurteil ersetzt wird. Wie dem auch sei, beim Kauf von Tickets eines Sportevents ist der Grad der Integrativität niedrig, weil beim Ticketkauf weder ein Eingriff auf viele Wertschöpfungsstufen erfolgt noch eine intensive Kommunikation mit dem Käufer notwendig ist. Zudem sind auch nur relativ wenige Ticketmerkmale kaufentscheidend, sodass auch aus dieser Perspektive einer Anwendung der Conjoint-Analyse für Kaufentscheidungen von Tickets für Sportevents nichts im Wege steht.

Die Individualität kann auf einem Kontinuum zwischen standardisierten und maßgeschneiderten Leistungsergebnissen dargestellt werden. Maßgeschneiderte Sportdienstleistungen (z.B. 
individuelle Trainings- oder Physiotherapiepläne sowie Investitionsberatungen für Profisportler) gehen dabei auf die individuellen Ansprüche, Wünsche und Bedürfnisse der Kunden ein. Oft sind diese bei Vertragsabschluss nur grob erfasst und werden erst im Laufe der Leistungserstellung näher definiert, sodass die Merkmale nach Vertragsabschluss noch stärker variieren können. Der Verkauf von Tickets eines Sportevents zeichnet sich durch eine geringe Individualität aus. Die aus dem Ticket abzuleitenden Ansprüche zur Leistungserstellung sind weitgehend standardisiert. Daher ist auch aus dieser Perspektive die Conjoint-Analyse für die Ermittlung von Preisbereitschaften von Tickets geeignet.

\section{Geringe Anzahl an Eigenschaften bestimmt die Kaufentscheidung vollständig}

Für die valide Durchführung einer Conjoint-Analyse ist es unerlässlich, dass die Kaufentscheidung durch eine möglichst geringe Anzahl an Eigenschaften bestimmt wird. In der Literatur wird diskutiert, dass zu viele Eigenschaften für die Ergebnisse der Befragung kontraproduktiv sein können. Dies ist zum einen auf die begrenzte menschliche Leistungsfähigkeit, zum anderen aber auch auf forschungsökonomische Gründe zurückzuführen (Weiber \& Mühlhaus, 2009). Auch wenn man durchaus reduzierte Designs anwenden kann, führt dennoch die Berücksichtigung einer $\mathrm{zu}$ hohen Anzahl an Stimuli zu einer Informationsüberflutung der Befragten (Green \& Srinivasan, 1990). Die Umfrage würde zu lange dauern und könnte aufgrund der Überforderung der Probanden auch zu einer hohen Abbruchquote führen. Dementsprechend ist es unerlässlich, sich auf wenige, dafür zentrale Eigenschaften zu konzentrieren. Auch die Anzahl der Eigenschaftsausprägungen muss berücksichtigt werden. Der Number-of-level-Effekt bedeutet, dass eine höhere Anzahl von Eigenschaftsausprägungen eine höhere relative Bedeutung für Eigenschaften mit mehr Ausprägungen nach sich zieht. Dies hat verzerrte Nutzenwerte zur Folge (Verlegh, Schif- ferstein, \& Wittink, 2002; Wittink, Huber, Zandan, \& Johnson, 1992).

Im Vergleich zu den Ausführungen von Woratschek $(1998,2000)$ ermöglichen seitdem entwickelte Varianten der Conjoint-Analyse die Berücksichtigung einer höheren Anzahl an Merkmalen. Diesen methodischen Weiterentwicklungen weisen auch eine höhere Validität auf(Heidbrink, 2008). Aus diesem Grund sollte die Anforderung der geringen Anzahl an Eigenschaften relativiert werden, wobei die Anzahl an Merkmalen in einem zweckmäßigen Forschungsdesign noch immer begrenzt ist.

Im Allgemeinen haben Tickets für Sportevents unterschiedliche Eigenschaften, die die Kaufentscheidung beeinflussen können. Dies kann beispielsweise die sportliche Relevanz (z. B. Ligaspiel vs. Playoffs), der Wochentag, der Startzeitpunkt, die gegnerische Mannschaft, die Sitzkategorie oder sogar der Veranstaltungsort sein. All diese Eigenschaften können die Präferenzen der Verbraucher beeinflussen. Wie jedoch schon diskutiert, führt eine zu hohe Anzahl an Eigenschaften zu einer Überforderung der Probanden. Dementsprechend sind eine möglichst geringe Anzahl und die sorgfältige Auswahl der relevanten Eigenschaften von zentraler Bedeutung. So sind Eigenschaften wie der Wochentag, die Startzeit und auch der Veranstaltungsort eines bestimmten Events zum Verkaufsstart der Eintrittskarten bekannt. Da diese im vorliegenden Fall vom veranstaltenden Verband in Abstimmung mit den übertragenden TV-Anstalten festgelegt werden, können sie nicht vom Ticketanbieter beeinflusst und realisiert werden. Zudem können bei wechselnden Anstoßtagen und -zeiten private oder berufliche Terminkonflikte zum Ausschluss bestimmter Eigenschaftsausprägungen führen. Dies würde zur Verletzung grundlegender Anforderungen an integrierte Merkmale einer Conjoint-Analyse führen (Backhaus et al., 2018). Bei Analysen im Ticketing sollten demnach diese Eigenschaften je nach Betrachtungsweise als konstant betrachtet werden. Eine Unterscheidung der Tickets für ein Sportevent findet regelmäßig auf Basis unterschiedlicher
Sitzkategorien und daraus resultierender unterschiedlicher Preise statt.

In dieser Studie gehen wir davon aus, dass der Ticketpreis, die Sitzkategorie und sportliche Relevanz die Kaufentscheidung signifikant determinieren. Insgesamt kann für Tickets eines Sportevents eine realistische Kaufentscheidung bereits mit einer geringen Anzahl an Eigenschaften modelliert werden, sodass eine Conjoint-Analyse mit hoher Validität durchgeführt werden kann.

\section{Hohes Involvement der Kunden}

Sportevents sind mit vielen Emotionen verbunden (Biscaia, Correia, Rosado, Maroco, \& Ross, 2012; Hanin, 1999). Dementsprechend ist auch die Identifikation mit Sportlern oder Sportteams bei Zuschauern oft stark ausgeprägt (Donavan, Carlson, \& Zimmermann, 2005; Ströbel, Woratschek, \& Durchholz, 2019; Sutton, McDonald, Milne, \& Cimperman, 1997; Wann \& Branscombe, 1990, 1993). Ein hohes Involvement der $\mathrm{Zu}$ schauer ist bei einem Kauf von Tickets für Sportevents also durchaus anzunehmen. Bei einem hohen Involvement kann man davon ausgehen, dass der in der Conjoint-Analyse unterstellte Trade-off von Produktmerkmalen gegeben ist.

\section{Keine Make-or-buy-Entscheidung}

Die Conjoint-Analyse bildet keine Make-or-buy-Entscheidung ab, wie sie sich häufig im Bereich von Dienstleistungen stellen kann. Ein Sportevent auf professionellem Niveau inklusive der Teilnahme beliebter Sportler oder Teams ist nahezu unmöglich nur durch die Eigenleistung des Kunden zu substituieren. Aus diesem Grund kann auch die Frage nach einer Make-or-buy-Entscheidung ausgeschlossen werden.

\section{Kosten unabhängig der Auslastung}

Für die Organisation eines Sportevents fallen hohe Fixkosten an (Masterman, 2014). Mietkosten für die Veranstaltungsstätte sowie die Personalkosten für die Sportler, Teams und weitere Akteure des sportlichen Wettkampfs fallen unabhängig von der Auslastung der Ver- 
Tab. 1 Merkmale und deren Eigenschaften im Conjoint-Forschungsdesign

Ticketpreis (€)

50,00

35,00

20,00
Sitzkategorie

Kategorie 1: Haupttribüne, Unterrang

Kategorie 2: Haupttribüne, Oberrang

Kategorie 3: Tribünen hinter den Toren
Spielkategorie

WM-Gruppenspiel

EM-Qualifikationsspiel

Freundschaftsspiel anstaltungsstätte an. Ebendies gilt auch für die Personalkosten rund um die Organisation des Events. Die variablen Kosten für einen einzelnen Zuschauer eines Sportevents sind daher äußerst gering und tendieren gegen null. Daher sind die anfallenden Kosten eines Sportevents zum größten Teil unabhängig von der Auslastung der Veranstaltungsstätte, sodass man für gewinnoptimale Preisentscheidungen die Kostenseite vernachlässigen kann. Die ConjointAnalyse ermöglicht die Modellierung von erlösoptimalen Preiskalkulationen. Daher ist eine weitere Betrachtung der Kostenseite nicht erforderlich.

\section{Konkurrenzverhalten}

Die Conjoint-Analyse wird häufig für Produktentwicklungen und -verbesserungen sowie zur Preisfindung eingesetzt. Dabei werden in der klassischen Conjoint-Analyse grundsätzlich keine Konkurrenzreaktionen modelliert. Eine Besonderheit des Sportmanagements ist jedoch die Tatsache, dass professionelle Sportvereine und -verbände als regionale „Quasi“-Monopolisten agieren. Studien zu Fanidentifikation und -loyalität zeigen eindrucksvoll, dass trotz schlechter Leistungen der Sportler oder Teams und der damit verbundenen Unzufriedenheit der Zuschauer und Fans ein Wechsel des Anbieters oder Klubs oder auch der Sportart nahezu ausgeschlossen ist (Woratschek, Horbel, \& Popp, 2018). So ist es nahezu undenkbar, dass ein unzufriedener Fan der deutschen Fußball-Nationalmannschaft dieser den Rücken kehrt, um die niederländische Nationalmannschaft zu unterstützen. Der Einbezug der Konkurrenzreaktionen im Ticketing für Sportevents ist somit vernachlässigbar.

Zusammenfassend lässt sich festhalten, dass die von Woratschek $(1998,2000)$ adressierten Anforderungen zum Einsatz der Conjoint-Analyse im Dienstleis- tungsbereich für das Dienstleistungsangebot des Ticketings für Sportevents weitgehend erfüllt werden. Somit ist die Conjoint-Analyse grundsätzlich für die gewinnmaximale Bestimmung von Ticketpreise gut geeignet.

\section{Empirische Untersuchung}

\section{Forschungsdesign}

Bei der Conjoint-Analyse ist die Konstruktion eines passenden Untersuchungsdesigns von entscheidender Bedeutung, um die Entscheidungsfindung der Probanden so realitätsnah wie möglich abzufragen. Die zentralen Bausteine einer Conjoint-Studie sind Produktkarten, die aus unterschiedlichen Handlungsalternativen mit definierten Eigenschaften und unterschiedlichen Ausprägungen bestehen (Baier \& Brusch, 2009b). In der nachfolgenden Studie bilden verschiedene Sporteventtickets mit variierenden Merkmalsausprägungen diese Produktkarten. Es ist davon auszugehen, dass die Preisbereitschaft unter anderem von leistungsbezogenen Merkmalen wie der Sitzkategorie abhängt. So ist grundsätzlich zu erwarten, dass $\mathrm{Zu}$ schauer für einen vermeintlich besseren Sitzplatz eine höhere Preisbereitschaft aufweisen, als für einen vermeintlich schlechteren Platz. Zudem ist im Fall von Fußballspielen der deutschen Frauen Fußball-Nationalmannschaft insbesondere auch die Eigenschaft „Spielkategorie“ von großer Bedeutung. Diese ist als Indikator für die sportliche Relevanz zu sehen. So werden in der Regel Freundschaftsspiele sowie Qualifikationsspiele zu Europa- oder Weltmeisterschaften ausgetragen. Im vorliegenden Fall werden diese um Gruppenspiele einer Weltmeisterschaft ergänzt, da diese in der Regel eine höhere sportliche Relevanz genießen als EM-Qualifikationsspiele. Es ist auch hier davon auszugehen, dass sportlich relevantere Spiele wie die ei- ner Weltmeisterschaft zu einem höheren Nutzen bei den Zuschauern führen als sportlich unbedeutendere Spiele, wie z. B. Freundschaftsspiele (Woratschek \& Schafmeister, 2008). Bei einer Kaufentscheidung spielt naturgemäß auch der Kaufpreis eine wichtige Rolle. Da die Erfahrungen zu den Preisbereitschaften der Zuschauer eines Spiels der deutschen Frauen Fußball-Nationalmannschaft nur eine relativ geringe Datenbasis aufweist und zudem die ermittelten Preis-Absatzfunktionen nicht konsistent sind, wurde der Ticketpreis ebenfalls in das Untersuchungsdesign mit aufgenommen.

Weitere Informationen zu den einzelnen Spielen stehen zum Erhebungszeitpunkt nicht zur Verfügung. So ist beispielsweise unbekannt, welche Spielpaarungen in welchem Stadion zu welcher Zeit stattfinden. Dementsprechend wurde ein fiktives Spiel zwischen Deutschland und Dänemark ausgewählt und als standardisierte Informationen in das Feldexperiment aufgenommen. Als Austragungsort wurde das Stadion in Frankfurt am Main ausgesucht, da in diesem auch die Befragung stattfand. Die Anstoßzeit wurde auf $18.15 \mathrm{Uhr}$ festgelegt, ein Wochentag jedoch nicht, um Terminkollisionen mit privaten Terminen $\mathrm{zu}$ vermeiden. Somit wurden 3 Hauptmerkmale und ihre Merkmalseigenschaften ausgewählt (• Tab. 1).

Im Rahmen der empirischen Untersuchung wird dem Ticketpreis eine Opferkomponente zugesprochen (Woratschek, 1998). Dementsprechend wird zwischen den Teilnutzenwerten des Preises ein negativ-linearer Zusammenhang (Backhaus et al., 2018, S. 535: „LINEAR LESS“) angenommen. Dies bedeutet, dass ein höherer Ticketpreis einen geringeren Nutzen zur Folge hat, wenn die weiteren Merkmale konstant bleiben. Im Gegensatz werden für die Merkmale Sitzkategorie und Spielkategorie keinerlei Annahmen über den Zusammenhang der Merkmalseigenschaften und dem daraus resultierenden Nutzen (Backhaus et al., 2018, S. 535: „DISCRETE“) getroffen.

Das Forschungsdesign besteht somit aus 3 Merkmalen mit jeweils 3 Eigenschaften, was insgesamt zu $3^{3}$, also 27 möglichen Ticketkombinationen führt. 
Hauptbeitrag

\begin{tabular}{llll}
\hline \multicolumn{3}{l}{ Tab. 2 } & Teilnutzenwerte und relative Wichtigkeiten der aggregierten Conjoint-Analyse \\
& Teilnutzenwert & Standardfehler & Relative Wichtigkeit \\
\hline Ticketpreis & & & $39,17 \%$ \\
\hline $50 €$ & $-3,782$ & 0,612 & - \\
$35 €$ & $-2,522$ & 0,408 & - \\
$20 €$ & $-1,261$ & 0,204 & - \\
\hline Spielkategorie & & & $41,65 \%$ \\
WM-Gruppenspiel & 1,290 & 0,236 & - \\
\hline EM-Qualifikationsspiel & 0,100 & 0,236 & - \\
\hline Freundschaftsspiel & $-1,390$ & 0,236 & - \\
\hline Sitzkategorie & & & $19,18 \%$ \\
Kat. 1 (rot) & 0,631 & 0,236 & - \\
Kat. 2 (gelb) & $-0,028$ & 0,236 & - \\
Kat. 3 (blau) & $-0,603$ & 0,236 & - \\
Konstante & 7,522 & 0,411 & -
\end{tabular}

Nach Addelman (1962) wurden daher diese 27 Kombinationen mit Hilfe eines lateinischen Quadrats auf 9 Ticketvarianten reduziert.

Um die Tickets so realistisch wie möglich zu gestalten, wurde das ursprüngliche Ticketdesign sowie die offiziellen Markenlogos von DFB, FIFA und UEFA verwendet. Entsprechend der Profilmethode sind alle Merkmale auf den Tickets vertreten (Teichert, 2000), sowohl schriftlich als auch visuell. Das fiktive Spiel zwischen Deutschland und Dänemark, die Anstoßzeit und der Austragungsort wurden auf jedem Ticket als konstante Information bereitgestellt.

- Abb. 1 zeigt exemplarisch 3 der verwendeten 9 Produktkarten.

Da das Ziel dieser empirischen Untersuchung die Ermittlung von Preisaufschlägen bzw. -abschlägen ist und Wert auf ein schlankes Forschungsdesign gelegt wurde, wurde auf eine LimitConjoint-Analyse (Voeth \& Schumacher, 2003) verzichtet.

\section{Datenerhebung}

Im Hinblick auf die Realisierbarkeit der Befragung ist insbesondere auf die Erreichbarkeit der Grundgesamtheit, Zuschauer von Spielen der deutschen Frauen Fußball-Nationalmannschaft, zu achten. Ein angemessener Rahmen hierfür stellt ein Länderspiel besagter Mannschaft dar, da dort genau die Personen erreicht werden, die sich zuvor für den Kauf eines Länderspieltickets ent- schieden haben. Aufgrund des schlank gewählten und reduzierten Forschungsdesigns ist auch die Überforderung der Probanden im hektischen Umfeld des Stadions weitestgehend eliminiert. Die Datenerhebung fand daher während eines Freundschaftsspiels zwischen den Frauen Fußball-Nationalmannschaften Deutschlands und Brasiliens am 22. April 2009 in der Frankfurter Commerzbank Arena statt. Die Befragung startete $3 \mathrm{~h}$ vor Beginn des Freundschaftsspiels innerhalb des Stadiongeländes und dauerte pro Proband ca. $5 \mathrm{~min}$. Insgesamt konnten 446 Zuschauer mittels ConvenienceSampling befragt werden. Die Datenbereinigung erfolgte zunächst im Hinblick auf 41 unvollständig ausgefüllte Fragebögen, da diese keine Schätzung von Teilnutzenwerten erlauben. Des Weiteren wurden 124 weitere Fälle aufgrund inkonsistenter Werte ausgeschlossen, da diese bei steigenden Preisen einen höheren Nutzen zeigten. Dies stand im Widerspruch mit der in der Studienkonzeption getroffenen Annahme, dass mit steigendem Ticketpreis der Nutzen der Probanden fällt. Insgesamt konnten demnach 281 Fragebögen für die weitere Analyse verwendet werden.

\section{Ergebnisse}

Die Ergebnisse der aggregierten Conjoint-Analyse sind in $\mathbf{0}$ Tab. 2 zu sehen. Durch die Analyse der Spannweiten der Teilnutzenwerte pro Merkmal kann auf die Wichtigkeit geschlossen werden.
Auf aggregierter Ebene weisen die befragten Zuschauer der Spielkategorie $44 \%$ relative Wichtigkeit zu, gefolgt vom Preis mit $31 \%$ und der Sitzkategorie mit $25 \%$. Aufgrund der Heterogenität von Sporteventbesuchern (Hunt et al., 1999; Mullin et al., 2014; D. Wann \& Branscombe, 1990) ist die aggregierte Analyse der gesammelten Daten grundsätzlich mit einem Informationsverlust verbunden. Die Zuschauer wurden daher entsprechend ihrer Präferenzen mittels einem hierarchisch-agglomerativen Clusterverfahren segmentiert, die in sich möglichst homogen sind. Um potenzielle Ausreißer zu identifizieren, wurde zunächst eine Single-Linkage-Clusteranalyse durchgeführt. Dabei wurden 3 Ausreißer identifiziert, die von der weiteren Analyse ausgeschlossen wurden. Anschließend folgte eine AverageLinkage-Clusteranalyse mit normalisierten Teilnutzenwerten und der PearsonKorrelation. Diesen Ansätzen folgend, wurde die Stichprobe zunächst auf eine endgültige Stichprobengröße von 278 Fällen reduziert und daraufhin 5 Cluster identifiziert. Diese Cluster unterscheiden sich hinsichtlich ihrer Präferenzstruktur deutlich voneinander. Sie werden in - Tab. 3 und im Folgenden genauer beschrieben. - Tab. 4 zeigt die zusätzlichen Preisbereitschaften der einzelnen Segmente für veränderte Ticketoptionen, die auf den Teilnutzenwerten aus - Tab. 3 resultieren. Diese gehen von den Ausprägungen mit dem geringsten Nutzen aus.

Das erste Cluster, preissensitive $\mathrm{Zu}$ schauer, hat eine Größe von 35,6\%. Für sie ist der Preis mit $58 \%$ für die Kaufentscheidung am wichtigsten. Entsprechend toleriert diese Gruppe nur geringe Aufschläge für eine bessere Spielkategorie oder einen besseren Platz. So erhöht sich die Preisbereitschaft lediglich um $4,21 €$ für ein WM-Gruppenspiel im Vergleich zu einem Freundschaftsspiel, bzw. um $4,84 €$ für Sitzkategorie $1 \mathrm{im}$ Vergleich zu Sitzkategorie 3. Es lässt sich also deutlich erkennen, dass dieses Cluster nicht bereit ist, für eine bessere Qualität hohe Preiszuschläge in Kauf zu nehmen und deshalb nach günstigen Tickets sucht.

Im Gegensatz dazu dominiert im zweiten Cluster relevanzorientierte $\mathrm{Zu}$ - 
Tab. 3 Teilnutzenwerte der identifizierten Cluster

\begin{tabular}{|c|c|c|c|c|c|}
\hline & $\begin{array}{l}\text { Preissensitive } \\
\text { Zuschauer }\end{array}$ & $\begin{array}{l}\text { Relevanzorientierte } \\
\text { Zuschauer }\end{array}$ & $\begin{array}{l}\text { Fanblock- } \\
\text { Zuschauer }\end{array}$ & $\begin{array}{l}\text { Erlebnisorientierte } \\
\text { EM-Fans }\end{array}$ & $\begin{array}{l}\text { Sitzplatzorientierte } \\
\text { Zuschauer }\end{array}$ \\
\hline Größe & $\begin{array}{l}35,6 \% \\
n=99\end{array}$ & $\begin{array}{l}48,9 \% \\
n=136\end{array}$ & $\begin{array}{l}3,6 \% \\
n=10\end{array}$ & $\begin{array}{l}2,5 \% \\
n=7\end{array}$ & $\begin{array}{l}9,4 \% \\
n=26\end{array}$ \\
\hline \multicolumn{6}{|l|}{ Ticketpreis } \\
\hline $50 €$ & $-7,136$ & $-1,893$ & $-2,500$ & $-1,429$ & $-2,019$ \\
\hline $35 €$ & $-4,758$ & 1,262 & $-1,667$ & $-0,952$ & $-1,346$ \\
\hline $20 €$ & $-2,379$ & $-0,631$ & $-0,833$ & $-0,476$ & $-0,673$ \\
\hline \multicolumn{6}{|l|}{ Spielkategorie } \\
\hline WM-Gruppenspiel & 0,273 & 2,446 & 0,233 & $-1,238$ & 0,205 \\
\hline EM-Qualifikationsspiel & 0,121 & $-0,002$ & $-0,467$ & 2,619 & 0,090 \\
\hline Freundschaftsspiel & $-0,394$ & $-2,444$ & 0,233 & $-1,381$ & $-0,295$ \\
\hline \multicolumn{6}{|l|}{ Sitzkategorie } \\
\hline Kat. 1 (rot) & 0,354 & 0,490 & 0,467 & 0,619 & 2,487 \\
\hline Kat. 2 (gelb) & 0,061 & 0,066 & $-2,200$ & $-0,905$ & 0,218 \\
\hline Kat. 3 (blau) & $-0,414$ & $-0,556$ & 1,733 & 0,286 & $-2,705$ \\
\hline Konstante & 9,758 & 6,262 & 6,667 & 5,952 & 6,346 \\
\hline \multicolumn{6}{|l|}{ Relative Wichtigkeiten } \\
\hline Ticketpreis & $58 \%$ & $15 \%$ & $20 \%$ & $12 \%$ & $16 \%$ \\
\hline Spielkategorie & $24 \%$ & $66 \%$ & $17 \%$ & $60 \%$ & $19 \%$ \\
\hline Sitzkategorie & $18 \%$ & $19 \%$ & $63 \%$ & $28 \%$ & $65 \%$ \\
\hline
\end{tabular}

Tab. 4 Zusätzliche Preisbereitschaften für andere Merkmalseigenschaften

\begin{tabular}{|c|c|c|c|c|c|}
\hline & $\begin{array}{l}\text { Preissensitive } \\
\text { Zuschauer }\end{array}$ & $\begin{array}{l}\text { Relevanzorientierte } \\
\text { Zuschauer }\end{array}$ & $\begin{array}{l}\text { Fanblock- } \\
\text { Zuschauer }\end{array}$ & $\begin{array}{l}\text { Erlebnisorientierte } \\
\text { EM-Fans }\end{array}$ & $\begin{array}{l}\text { Sitzplatzorientierte } \\
\text { Zuschauer }\end{array}$ \\
\hline \multicolumn{6}{|l|}{ Spielkategorie } \\
\hline WM-Gruppenspiel & $+4,21 €$ & $+116,21 €$ & $+0,00 €$ & $+4,50 €$ & $+11,14 €$ \\
\hline EM-Qualifikationsspiel & $+3,25 €$ & $+58,02 €$ & $-12,60 €$ & $+126,00 €$ & $+8,57 €$ \\
\hline Freundschaftsspiel & - & - & - & - & - \\
\hline \multicolumn{6}{|l|}{ Sitzkategorie } \\
\hline Kat. 1 (rot) & $+4,84 €$ & $+24,88 €$ & $-22,80 €$ & $+10,50 €$ & $+115,71 €$ \\
\hline Kat. 2 (gelb) & $+2,99 €$ & $+14,80 €$ & $-70,80 €$ & $-37,50 €$ & $+65,14 €$ \\
\hline Kat. 3 (blau) & - & - & _- & - & _- \\
\hline
\end{tabular}

schauer, das mit 136 Probanden (48,92\%) das größte Segment darstellt, das Merkmal Spielkategorie die Präferenzen. Dabei sind große Preissprünge zwischen den unterschiedlichen Spielkategorien festzustellen. So erhöht sich die Preisbereitschaft für ein WM-Gruppenspiel um $116,21 €$ im Vergleich zu einem Freundschaftsspiel. Der Preis spielt mit 15\% relativer Wichtigkeit nur eine untergeordnete Rolle. Es zeigt sich also deutlich, dass für Gruppenspiele bei Weltmeisterschaften für relevanzorientierte $\mathrm{Zu}$ schauer deutlich höhere Ticketpreise durchgesetzt werden können, als diese im Frauenfußball bislang üblich waren. Wenn man die üblichen Ticketpreise deutschen Bundesliga-Spielen in der günstigsten Sitzkategorie um die $15 €$ zugrunde legt, kann die Relevanz eines Spiels zu einem sieben bis achtmal höheren Preis in diesem Zuschauersegment führen.

Für Zuschauer in Cluster 3 ist die Sitzkategorie mit $63 \%$ am wichtigsten. Dabei fällt aber auf, dass die Kategorie 3 (hinter dem Tor) am meisten präferiert wird, gefolgt von Kategorie 1. Es ist anzunehmen, dass Zuschauer dieses Clusters einen Platz in den Fankurven bevorzugen, die üblicherweise hinter dem Tor zu finden sind. Entsprechend kann man dieses Cluster als Fanblock-Zuschauer be- zeichnen. Das Cluster ist mit 3,6\% der Gesamtstichprobe sehr klein.

Ein weiteres sehr kleines Cluster wurde als erlebnisorientierte EM-Fans identifiziert. Wie für relevanzorientierte $\mathrm{Zu}$ schauer in Cluster 2, ist die Spielkategorie mit $60 \%$ am wichtigsten, gefolgt von der Ticketkategorie mit $28 \%$. In den Präferenzen innerhalb der Spielkategorie unterscheiden sich die Cluster jedoch massiv. So schätzen erlebnisorientierte EM-Fans EM-Qualifikationsspiele am meisten. Darüber hinaus werden Plätze in Kategorie 3 denen in Kategorie 2 bevorzugt. Für ein WM-Gruppenspiel steigt die Preisbereitschaft gegen- 
über einem Freundschaftsspiel nur um $4,50 €$.

Im fünften Cluster, den sitzplatzorientierten Zuschauern, dominiert die Sitzkategorie mit einer relativen Wichtigkeit von $65 \%$. Dabei sind sitzplatzorientierte Zuschauer bereit, einen Aufpreis von $65,14 € \mathrm{zu}$ bezahlen, um in Kategorie 2 anstatt Kategorie $3 \mathrm{zu}$ sitzen. Für einen Platz in Kategorie 1 würden sie sogar einen Aufpreis von 115,71€ im Vergleich zu Kategorie 3 in Kauf nehmen. Der Preis spielt mit $16 \%$ relativer Wichtigkeit eine Nebenrolle. Mit 9,35\% ist dieses das drittgrößte der identifizierten Cluster.

\section{Diskussion und Implikationen}

Die Präferenzen der identifizierten Cluster unterscheiden sich deutlich, sowohl untereinander als auch hinsichtlich der Ergebnisse auf aggregierter Ebene. Es zeigt sich also, dass eine Segmentierung von Zuschauern anhand ihrer Ticketpräferenzen zielführend ist. Um die Nachfrage aller Zuschauer zu befriedigen, ist es unerlässlich, die Preisstrategie an den Präferenzen der einzelnen Segmente auszurichten. Basierend auf den tatsächlichen Ticketpreisen des untersuchten Freundschaftsspiels können somit konkrete Ticketpreise für andere Wettbewerbe, wie in diesem Fall EMQualifikationsspiele oder WM-Gruppenspiele, festgelegt werden. Darüber hinaus können die Preise innerhalb einer Veranstaltung an die Größe der einzelnen Segmente sowie deren Präferenzen angepasst und somit eine den Präferenzen der Zuschauer angepasste Preisdiskriminierung angestrebt werden. Aus Gründen der Umsetzbarkeit und zur Ableitung von Handlungsempfehlungen für Sportmanager empfehlen wir jedoch, diese 5 Cluster-Lösung mit zwei äußerst kleinen Segmenten (mit 3,6 und 2,5\% aller Probanden) zu 3 Hauptzuschauersegmenten mit substanziell unterschiedlichen Präferenzen zu aggregieren: sitzkomfortorientierte Zuschauer ( $13 \%$, Cluster 3 und 5), preissensitive $\mathrm{Zu}$ schauer (36\%, Cluster 1), und relevanzorientierte Zuschauer (51\%, Cluster 2 und 4).

Im Fall eines repräsentativen Samples sollten somit ca. $10 \%$ der besten Sitzplät- ze im Stadion für sitzkomfortorientierte Zuschauer vorgesehen werden, da dieses Segment bereit ist, einen hohen Preisaufschlag für die besten Sitzplätze in Kauf zu nehmen. Manager sollten daher auf eine angemessene Zahl qualitativ hochwertiger und hochpreisiger Plätze achten. $\mathrm{Zu}$ viele Plätze in dieser Kategorie würden zu einem Überangebot an Tickets mit einem hohen Preis führen, den andere Zuschauer jedoch nicht bereit sind $\mathrm{zu}$ bezahlen. $\mathrm{Zu}$ wenige Plätze in dieser Kategorie bedeuten einen Verzicht auf zusätzliche Einnahmen.

Die Studie zeigt auch, dass nicht alle Zuschauersegmente die Sitzqualität als besonders relevant einstufen. Für über ein Drittel der Befragten ist der Preis entscheidend. Dabei spielt es für diese preissensitiven Fans nur eine untergeordnete Rolle, in welcher Kategorie des Stadions der Platz ist. Daraus lässt sich ableiten, dass ca. ein Drittel der Tickets zu einem günstigen Preis angeboten werden sollten, um dieses Zuschauersegment nicht zu verärgern.

Dem dritten Segment der spielorientierten Zuschauer ist ein Spiel mit hoher sportlicher Relevanz besonders wichtig. Dies spiegelt sich in den höheren Nutzenwerten von WM-Gruppenspielen im Vergleich zu EM-Qualifikationsspielen und Freundschaftsspielen wider. Für mehr als die Hälfte aller Probanden ist demnach die sportliche Relevanz maßgeblich für die Kaufentscheidung eines Sporteventtickets. Dabei nehmen diese auch einen erheblichen Preisaufschlag in Kauf, sodass Zuschläge für Events mit erhöhter sportlicher Relevanz auf die Akzeptanz von vielen Zuschauern stoßen. Wenn man bedenkt, dass die sportliche Relevanz unter bestimmten Umständen zu einer sieben bis achtmal höheren Preisbereitschaft in bestimmten Zuschauersegmenten führt, wird deutlich, dass zusätzliche Forschungen zur Gestaltung von präferenzorientierten Ticketpreisen von Sportevents mit Hilfe von Conjoint-Analysen dringend angezeigt sind. Die vorliegende Analyse liefert daher wertvolle Informationen für die Preispolitik und die zukünftigen Marketingaktivitäten und kann u. a. dazu beitragen, dass Fanproteste aufgrund von Preisentscheidungen vermieden werden.

\section{Fazit}

Ziel dieser Studie war es, zunächst die Anwendbarkeit der Conjoint-Analyse zur Ermittlung von Präferenzen und Preisbereitschaften für den Kauf von Tickets im Sport zu diskutieren. Die grundsätzliche Eignung der Conjoint-Analyse in diesem Bereich wurde anhand von aus der Literatur entnommenen Anforderungskriterien festgestellt. Die Anwendung der Conjoint-Analyse ist für die präferenzorientierte Ticketpreisgestaltung insbesondere dann zu empfehlen, wenn es keine zuverlässigen und belastbaren historischen Daten zum Kaufverhalten der Zuschauer gibt. Darüber hinaus wurden in der vorliegenden Studie vor allem auf die sportliche Relevanz abgestellt, mit dem Ergebnis, dass neben den Sitzkategorien erhebliche Aufschläge für Sportevents mit erhöhter sportlicher Relevanz möglich sind. Es konnte herausgestellt werden, dass die Berücksichtigung von heterogenen Ticketpräferenzen bei Zuschauern unentbehrlich ist, da die Preisbereitschaften erheblich von den offerierten Sitzkategorien und der sportlichen Relevanz abhängen. Ein erheblicher Anteil der Zuschauer ist durchaus bereit, erhebliche Preiszuschläge für Sportevents mit hoher sportlicher Relevanz zu bezahlen. Nicht alle Fans sind preissensitiv, d.h. sie reagieren auf Preiserhöhungen mit Protesten oder Nichtkauf. Es gibt zudem $\mathrm{Zu}$ schauer, die bereit sind für gute Sitzplätze größere Preisaufschläge zu bezahlen.

Daher würde die Analyse aggregierter Daten zu verzerrten Ergebnissen und somit falschen Entscheidungen bezüglich der Preisgestaltung und der Bedarfsprognose von Sporteventtickets führen. Dementsprechend ist eine Clusteranalyse essenziell, um relevante Implikationen für Sportmanager abzuleiten, die den heterogenen Präferenzen der Zuschauer entsprechen. Demzufolge müssen die Ergebnisse bisheriger Studien zu Ticketpreisen, die von homogenen Ticketqualitäten (Heilmann \& Wendling, 1976; Rascher, McEvoy, Nagel, \& Brown, 2007) bzw. homogenen Präferenzen bzw. durchschnittlichen Preisbereitschaften für Tickets ausgehen (Bjørnskov Pedersen, Kiil, \& Kjær, 2011; Gershenfeld, 
2015; Greenwell, Popp, Brownlee, \& Jordan, 2007) kritisch hinterfragt werden.

Als Grundgesamtheit der vorliegenden Studie wurden die Zuschauer von Spielen der deutschen Frauen FußballNationalmannschaft definiert. Aussagen über potenzielle Zuschauer, die sich gegen einen Ticketkauf entschieden haben, sind demzufolge nicht möglich. In dieser Studie wurden weder erlösmaximale Preise für konkrete Sportevents in unterschiedlichen Kontexten (z.B. unterschiedliche Sportarten, unterschiedliche Ligen) noch konkrete Ticketpreise ermittelt. Für die Ermittlung von Preisbereitschaften für Tickets sind Weiterentwicklungen wie die Limit-Conjoint-Analyse $\mathrm{zu}$ verwenden (Voeth \& Hahn, 1998). Eine weitere signifikante Weiterentwicklung ist die Choice-based Conjoint-Analyse, in welcher Auswahlentscheidungen zwischen verschiedenen Ticketalternativen getroffen werden (Balderjahn, Hedergott, \& Peyer, 2009; DeSarbo, Ramaswamy, \& Cohen, 1995). Diese kann auch durch ein latentes Clusterverfahren erweitert werden, um der Heterogenität von Sportzuschauern noch besser gerecht zu werden (DeSarbo, Wedel, Vriens, \& Ramaswamy, 1992). Durch die Möglichkeit der Aufnahme weiterer Eigenschaften und Merkmalsausprägungen kann eine Kaufentscheidung noch realitätsnäher modelliert werden. (Green, Krieger, \& Wind, 2001). Dennoch bleibt die Anwendung der Conjoint-Analyse für die Modellierung von Kaufentscheidungen bei Tickets von Sportevents grundsätzlich sehr gut geeignet. Zukünftige Forschung im Sportmanagement sollte sich verstärkt mit methodischen Aspekten von Conjoint-Analysen auseinandersetzen, um konkrete Preisbereitschaften für unterschiedliche Ticket-Alternativen $\mathrm{zu}$ untersuchen. Dadurch können praxisrelevante Fragestellungen zur sinnvollen Gestaltung von Ticketkategorien im Sport und zur Gewährleistung höherer Einnahmen aus dem Verkauf von Tickets in unterschiedlichen Kontexten erforscht werden.

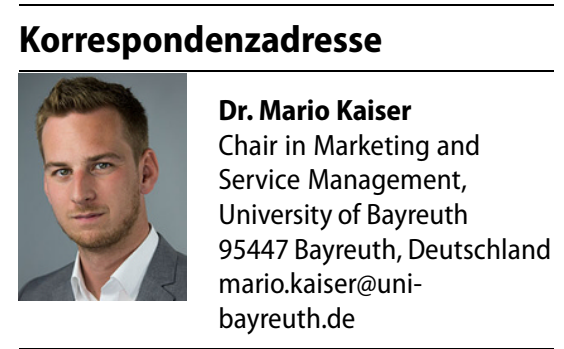

Funding. Open Access funding provided by Projekt DEAL.

\section{Einhaltung ethischer Richtlinien}

Interessenkonflikt. H. Woratschek, M. Kaiser, C. Durchholz und T. Ströbel geben an, dass kein Interessenkonflikt besteht.

Für diesen Beitrag wurden von den Autoren keine Studien an Menschen oder Tieren durchgeführt. Für die aufgeführten Studien gelten die jeweils dort angegebenen ethischen Richtlinien.

Open Access. Dieser Artikel wird unter der Creative Commons Namensnennung 4.0 International Lizenz veröffentlicht, welche die Nutzung, Vervielfältigung, Bearbeitung, Verbreitung und Wiedergabe in jeglichem Medium und Format erlaubt, sofern Sie den/die ursprünglichen Autor(en) und die Quelle ordnungsgemäßnennen, einen Link zur Creative Commons Lizenz beifügen und angeben, ob Änderungen vorgenommen wurden.

Die in diesem Artikel enthaltenen Bilder und sonstiges Drittmaterial unterliegen ebenfalls der genannten Creative Commons Lizenz, sofern sich aus der Abbildungslegende nichts anderes ergibt. Sofern das betreffende Material nicht unter der genannten Creative Commons Lizenz steht und die betreffende Handlung nicht nach gesetzlichen Vorschriften erlaubt ist, ist für die oben aufgeführten Weiterverwendungen des $\mathrm{Ma}$ terials die Einwilligung des jeweiligen Rechteinhabers einzuholen.

Weitere Details zur Lizenz entnehmen Sie bitte der Lizenzinformation auf http://creativecommons.org/ licenses/by/4.0/deed.de.

\section{Literatur}

Addelman, S. (1962). Orthogonal main-effect plans for asymmetrical factorial experiments. Technometrics, 4(1), 21-46.

Backhaus, K., Erichson, B., Plinke, W., \& Weiber, R. (2018). Multivariate Analysemethoden: Eine anwendungsorientierte Einführung. Bd. 15. Berlin, Heidelberg: Springer Gabler.

Baier, D., \& Brusch, M. (2009a). Erfassung von Kundenpräferenzen für Produkte und Dienstleistungen In D. Baier \& M. Brusch (Hrsg.), Conjointanalyse (S.3-17). Berlin, Heidelberg: Springer.

Baier, D., \& Brusch, M. (2009b). Konstruktion von Erhebungsdesigns bei der Conjointanalyse. In D. Baier \& M. Brusch (Hrsg.), Conjointanalyse (S. 73-82). Berlin, Heidelberg: Springer.

Balderjahn, I., Hedergott, D., \& Peyer, M. (2009). Choicebased conjointanalyse. In D. Baier \& M. Brusch
(Hrsg.), Conjointanalyse (Bd. 4, S. 129-146). Berlin, Heidelberg: Springer. https://doi.org/10. 1007/978-3-642-00754-5 9.

Biscaia, R., Correia, A., Rosado, A., Maroco, J., \& Ross, S. (2012). The effects of emotions on football spectators' satisfaction and behavioural intentions. European Sport Management Quarterly, 12(3), 227-242. https://doi.org/10.1080/16184742. 2012.679949.

Bjørnskov Pedersen, L., Kiil, A., \& Kjær, T. (2011). Soccer attendees' preferences for facilities at the Fionia park stadium: an application of the discrete choice experiment. Journal of Sports Economics, 12(2), 179-199. https://doi.org/10. $1177 / 1527002510379206$.

Deloitte (2015). Finanzreport deutscher Profisportligen 2015: Neue Impulse

DeSarbo, W.S., Ramaswamy, V., \& Cohen, S.H. (1995). Market segmentation with choicebased conjoint analysis. Marketing Letters, 6(2), 137-147.https://doi.org/10.1007/BF00994929.

DeSarbo, W.S., Wedel, M., Vriens, M., \& Ramaswamy, V. (1992). Latent class metric conjoint analysis. Marketing Letters, 3(3), 273-288. https://doi.org/ 10.1007/BF00994135.

DFB (2009). WM 2011: So funktioniert der Ticketverkauf. https://web.archive.org/ web/20091101125532/http:/www.dfb.de/ index.php?id $=500014 \&$ tx_dfbnews_pi 1 $\% 5$ BshowUid\%5D=20454\&tx_dfbnews_pi4 $\% 5 B$ cat $\% 5 D=167$. Zugegriffen: 25 . Juni 2019.

DFL Deutsche Fußball Liga GmbH (2019). Wirtschaftsreport 2019. https://www.dfl.de/de/dfl_ wirtschaftsreport_2019_de_m-2/.Zugegriffen: 25. Juni 2019

Donavan, D. T., Carlson, B. D., \&Zimmermann, M. (2005). The influence of personality traits on sports fan identification. Sport Marketing Quarterly, 14(1), 31-42.

Engelhardt, W.H., Kleinaltenkamp, M., \& Reckenfelderbäumer, M. (1993). Leistungsbündel als Absatzobjekte: Ein Ansatz zur Überwindung der Dichotomie von Sach- und Dienstleistungen. Zeitschrift für betriebswirtschaftliche Forschung, 45(5), 395-426.

Fiedler, H., Kaltenborn, T., Lanwehr, R., \& Melles, T. (2017). Conjoint-Analyse (2. Aufl.). Sozialwissenschaftliche Forschungsmethoden, Bd. 7. Augsburg: Rainer Hampp.

Gershenfeld, G. (2015). Conjoint analysis for ticket offerings at the Cleveland Indians. Interfaces, 45(2), 166-174. https://doi.org/10.1287/inte. 2014.0770

Green, P. E., \& Rao, V. R. (1971). Conjoint measurement for quantifying judgmental data. Journal of Marketing Research, 8(3), 355-363.

Green, P. E., \& Srinivasan, V. (1990). Conjoint analysis in marketing:new developments with implications for research and practice. Journal of Marketing, 54(4), 3-19. https://doi.org/10.2307/1251756.

Green, P. E., Krieger, A. M., \&Wind, Y. (2001). Thirtyyears of conjoint analysis: reflections and prospects. Interfaces, 31(3),56-73.https://doi.org/10.1287/ inte.31.3s.56.9676.

Greenwell, C., Popp, N., Brownlee, E., \& Jordan, J.S. (2007). Customer preference and student tickets: using conjoint analysis to develop ticket policy. International Journal of Sport Management, 8(3), 280-294.

Hanin, L. (1999). Emotions in sport. Champaign:Human Kinetics.

Heidbrink, M. (2008). Reliabilität und Validität von Verfahren der Präferenzmessung. Ein meta-analytischer Vergleich verschiedener Verfahren der 
Conjoint-Analyse. Saarbrücken: VDM-Verl. Dr. Müller.

Heilmann, R. L., \& Wendling, W. R. (1976). A note on optimum pricing strategies for sport events. In R. E. Machol, S. P. Ladany \& D. G. Morrison (Hrsg.), Management science in sports (S. 91-101). New York: North-Holland.

Hunt, K.A., Bristol, T., \& Bashaw, R.E. (1999). A conceptual approach to classifying sports fans. Journal of Services Marketing, 13(6), 439-452. https://doi.org/10.1108/08876049910298720.

Johnson, R. M. (1974). Trade-off analysis of consumer values. Journal of Marketing Research, 11(2), 121-127.

Lee, J., \& Ferreira, M. (2011). Cause-related marketing: the role of team identification in consumer choice of team licensed products. Sport Marketing Quarterly, 20(3), 157-169.

Leigh, T.W., MacKay, D. B., \& Summers, J. O. (1984). Reliability and validity of conjoint analysis and self-explicated weights: a comparison. Journal of Marketing Research, 21(4), 456-462. https://doi. org/10.1177/002224378402100410.

Masterman, G. (2014). Strategic sports event management (3. Aufl.). New York: Routledge.

Mühlbacher, H., \& Botschen, G. (1990). BenefitSegmentierung von Dienstleistungsmärkten. Marketing: Zeitschrift Für Forschung Und Praxis, 12(3), 159-168.

Mullin, B. J., Hardy, S., \& Sutton, W. A. (2014). Sport marketing (4. Aufl.). Champaing, IL [u. a.]: Human Kinetics.

Rascher, D. A., McEvoy, C. D., Nagel, M. S., \& Brown, M. T. (2007). Variable ticket pricing in major league baseball. Journal of Sport Management, 21(3), 407-437. https://doi.org/10.1123/jsm.21.3.407.

Röckenhaus, F. (2012). „Kein Zwanni für 'nen Steher": Dortmund-Fans protestieren in Hamburg. https://www.sueddeutsche.de/sport/ dortmund-fans-protesieren-in-hamburg-keinzwanni-fuer-nen-steher-1.1263376. Zugegriffen:25. Juni 2019.

Ströbel, T., Woratschek, H., \& Durchholz, C. (2019). Clothes make the fan: the effect of team merchandise usage on team identification, fan satisfaction and team loyalty. Journal of Global Sport Management, 72(3), 1-18. https://doi.org/ 10.1080/24704067.2018.1531354.

Sutton, W. A., McDonald, M.A., Milne, G.R., \& Cimperman, J. (1997). Creating and fostering fan identification on professional sports. Sport Marketing Quarterly, 6(1), 15-22.

Teichert, T. (2000). Conjoint-Analyse. In A. Herrmann \& C. Homburg (Hrsg.), Lehrbuch. Marktforschung: Methoden, Anwendungen, Praxisbeispiele (2.Aufl. S. 471-512). Wiesbaden: Gabler.

Theysohn, S., Hinz, O., Nosworthy, S., \& Kirchner, M. (2009). Official supporters clubs: the untapped potential of fan loyalty. International Journal of Sports Marketing and Sponsorship, 10(4), 33-55. https://doi.org/10.1108/IJSMS-10-04-2009B004.

Verlegh, P.W. J., Schifferstein, H. N. J., \& Wittink, D. R (2002). Range and number-of-levels effects in derived and stated measures of attribute importance. Marketing Letters, 13(1), 41-52. https://doi.org/10.1023/A:1015063125062.

Voeth, M., \& Hahn, C. (1998). Limit conjoint-analyse. Marketing ZFP, 20(2), 119-132. https://doi.org/ 10.15358/0344-1369-1998-2-119.

Voeth, M., \& Schumacher, A. (2003). Ticket-Pricing für die WM 2006: Empfehlungen auf Basis von Informationen zur Zahlungsbereitschaft der deut- schen Bevölkerung. Hohenheimer Arbeits- und Projektberichte zum Marketing, Bd. 9.

Walker, M., \& Mondello, M. J. (2007). Moving beyond economic impact: a closer look at the contingent valuation method. International Journal of Sport Finance, 2(3), 149-160.

Wann, D. L., \& Branscombe, N. R. (1990). Die-hard and fair-weather fans: effects of identification on BIRGing and CORFing tendencies. Journal of Sport and Social issues, 14(2), 103-117.

Wann, D. L., \& Branscombe, N. R. (1993). Sports fans: measuring degree of identification with their team. International journal of sport psychology, 24(1), 1-17.

Weiber, R., \& Mühlhaus, D. (2009). Auswahl von Eigenschaften und Ausprägungen bei der Conjointanalyse. In D. Baier \& M. Brusch (Hrsg.) Conjointanalyse (S. 43-58). Berlin, Heidelberg: Springer.

Wittink, D. R., Huber, J., Zandan, P., \& Johnson, R. M. (1992). The number of levels effect in conjoint: where does it come from and can it be eliminated? http://www.sawtoothsoftware.com/ support/technical-papers/general-conjointanalysis/the-number-of-levels-effect-inconjoint-where-does-it-come-from-and-canit-be-eliminated-1992. Zugegriffen: 25. Juni 2019.

Woratschek, H. (1998). Preisbestimmung von Dienstleistungen: Markt- und nutzenorientierte Ansätze im Vergleich (1. Aufl.). Frankfurt am Main: Dt. Fachverl.

Woratschek, H. (2000). Conjoint Measurement - ein Verfahren zur nachfrageorientierten Preisbestimmung. In M.-P. Büch \& E. Franck (Hrsg.), Märkte und Organisationen im Sport: Institutionenökonomische Ansätze; Arbeitskreis Sportöko nomie ... Beiträge seiner Bayreuther Sitzung vom Mai 1998. Sportökonomie:, Bd. 1. Schorndorf: Hofmann.

Woratschek, H., \& Schafmeister, G. (2008). Einflussfaktoren der TV-Nachfrage von Sportübertragungen: Wettbewerb, Konsumkapital, Popularität, Spannungsgrad und Relevanz. In T. Schauerte (Hrsg.), Die Ökonomie des Sports in den Medien 2. Aufl. Sportkommunikation, (Bd. 1, S. 61-82). Köln: Von Halem.

Woratschek, H., Horbel, C., \& Popp, B. (2018). Videographic analysis of "weird guys": what do relationships mean to football fans? In J. J.Zhang \& B. G. Pitts (Hrsg.), The global football industry: marketing perspectives. World association for sport management series, (Bd. 3, S. 210-236). London: Routledge. 